\title{
Hypopituitarism due to central nervous system germinoma: a case report
}

\author{
DoThi Thanh Mai, Vu Chi Dung, Bui Phuong Thao, Nguyen Ngoc Khanh, Can Thi Bich Ngoc, Nguyen Phu Dat
}

From 8th APPES Biennial Scientific Meeting

Darwin, Australia. 29 October - 1 November 2014

Germ cell tumors in the central nervous system affect children and adults, the peak incidence is from $10-19$ years of age. Clinical presentation is mainly involving to the location and size of the tumor and the patient age. Endocrine abnormalities are the most common symptom.

We presented a case who had hypopituitarism due to central nervous system Germinoma.

A 10 years old girl was admitted to our hospital due to polyuria . Past history : she was diagnosed with diabetes insipidus and treated with oral minirin (desmopressin) 3 years ago. But she did not taken oral minirin for 2 years ago.

Physical examination showed : polyuria $(8 \mathrm{ml} / \mathrm{kg} / \mathrm{h})$, polydipsia, no dehydration, no weight gain. Her height was $131 \mathrm{~cm}(-0.5 \mathrm{SD})$. Her weight was $41 \mathrm{~kg}$. Her BMI was 23.9 (> 97th). She had no manifestation of puberty. Laboratory evaluation revealed : blood osmotic pressure : $304 \mathrm{mosm} / \mathrm{kg}$ (normal range : $275 \mathrm{mosm} / \mathrm{kg}-295$ $\operatorname{mosm} / \mathrm{kg}$ ), urinary osmotic pressure : $80 \mathrm{mosm} / \mathrm{kg}$ (normal range : $500-1200 \mathrm{mosm} / \mathrm{kg}$ ); serum cortisol at 8 a. $\mathrm{m}: 9.2 \mathrm{nmol} / \mathrm{l}$ (normal range : 200 - $600 \mathrm{moll} / \mathrm{l}$ ); plasma glucose level and electrolyte were normal; $\mathrm{T} 4$ : $38.8 \mathrm{nmo} / \mathrm{l}$ (normal : $50-150 \mathrm{nmol} / \mathrm{l}$ ), T3 : $2.35 \mathrm{nmol} / \mathrm{l}$ (normal range : $1-3 \mathrm{nmol} / \mathrm{l}$ ), TSH : $4.1 \mathrm{mcUl} / \mathrm{ml}$ (normai range : $1-5 \mathrm{mcUI} / \mathrm{ml}$ ); $\mathrm{LH}: 0.16 \mathrm{UI} / \mathrm{ml}$ (normal range : 0,5 - 9,9 UI/l), FSH : $0.9 \mathrm{UI} / \mathrm{ml}$ (normal range : $1,4-5,6 \mathrm{UI} / \mathrm{l}$ ), Estradiol $<43.3 \mathrm{pmol} / \mathrm{l}$ (normal range : $97-169 \mathrm{pmol} / \mathrm{l}$ ). An MRI of brain showed: an under hypothalamic mass measuring $14 \times 20 \mathrm{~mm}$, suspected Germinoma.

She was treated with hormone replacement (desmopressin, levothyroxine, hydrocortisone) and radiation therapy.

Written informed consent was obtained from the patient's parent or guardian for publication of this Case

National Hospital of Pediatrics, Hanoi, Vietnam report (and any accompanying images). A copy of the written consent is available for review by the Editor-inChief of this journal.

Published: 28 April 2015

\section{doi:10.1186/1687-9856-2015-S1-P118}

Cite this article as: Mai et al:: Hypopituitarism due to central nervous system germinoma: a case report. International Journal of Pediatric Endocrinology 2015 2015(Suppl 1):P118.
Submit your next manuscript to BioMed Central and take full advantage of:

- Convenient online submission

- Thorough peer review

- No space constraints or color figure charges

- Immediate publication on acceptance

- Inclusion in PubMed, CAS, Scopus and Google Scholar

- Research which is freely available for redistribution
() Biomed Central 